\title{
FAMILY INVOLVEMENT IN THE REHABILITATION OF A STROKE PATIENT - (A CASE REPORT)
}

\author{
Journal website at; \\ http://mrtbjournal.org/index.php/njmr/issue/current/showToc \\ JANESKE VANDER WALDT ${ }^{1}$, JOYCE MOTHABENG ${ }^{1}$ \\ 'Physiotherapy Department, \\ University of Pretoria, South Africa \\ Correspondence to: \\ Joyce Mothabeng \\ Joyce.mothabeng@up.ac.za
}

\begin{abstract}
SUMMARY
Background and purpose: Family involvement is important for the successful rehabilitation outcome of a stroke patient. Very little has been published on this positive aspect, with most of the published literature focussing on the negative effects of a stroke on family functioning (i.e. caregiver burden). This retrospective case report highlights the importance of family participation in the rehabilitation of a stroke patient.
\end{abstract}

Case description: A 72 year old female patient with right hemiplegia is the subject of the report. The Barthel Index (BI) was used to evaluate the patient's functional abilities. Her main problem was the inability to plan functional activities.

Intervention: An inpatient rehabilitation programme was designed for the patient with contributions from the multidisciplinary team, including the family. The patient followed a routine daily program, receiving physiotherapy in the mornings and repeating the same activities in afternoon with her family assisting her. She received 10 treatment(s) sessions as an inpatient and four as outpatient.

Outcomes: Her BI score improved from 15 to 65 points and the self expressed short term goals were achieved by the end of her rehabilitation.

Conclusion: Early intervention and family support played a major role in the functional outcome of this patient, supporting the importance of family involvement in stroke rehabilitation.

Key words: Stroke, Family, Rehabilitation, Functional outcome, early intervention

\section{INTRODUCTION}

Stroke is the one of most common disabling disease on the world, with enormous psychosocial impact on both the patients and the family members (Teraoka \& Burgard 1992; Kotila, Numminen, Waltimo \& Kaste 1998; Visser et al, 2006). Following a stroke, role changes occur and family members find themselves in unfamiliar territory, in terms of performing unusual roles. The role changes in the family may be dramatic such that the caregiver (CG) may feel resentment toward the patient and the family may feel resentment toward the patient. This is particularly the case when the patient is immobile and incapable to perform basic activities of daily living -ADL. (Evans, Hendricks, Haselkorn, Bishop \& Baldwin, 1992; Umphred, 2001). Between 25 and $74 \%$ of stroke survivors require help with ADL (activities of daily living) from their caregivers due to the resultant disabilities of a stroke (Karla et al, 2004). In this part of the world, these caregivers are usually family members. Family involvement in the rehabilitation of patients with stroke is receiving increasing attention in literatures (Fritz and Penn, 1994; Karla et al, 2004, Patel et al 2004, White et al, 2004; Visser et al, 2008). Some studies have demonstrated objective evidence of positive family influence in stroke rehabilitation (Evans et al, 1992, Galvin, Cusack and Stokes, 2008). It is therefore important to prepare the immediate family for living with a patient with 
stroke.

The family of the patient presented in this case report was exceptionally involved in her rehabilitation from the first day of rehabilitation. The authors therefore decided to present this retrospective case to highlight the importance of family participation and their role in the functional outcome of a stroke patient.

\section{PATIENT HISTORY}

Mrs X is a 72 year old lady who sustained a stroke resulting in right hemiplegia and was admitted to a secondary level state hospital in South Africa on the same day. Her medical risk factors included diabetes mellitus, hypertension and chronic atrial fibrillation. She was started on antihypertensive, anticoagulant and diabetic medications and was referred to physiotherapy three days later.

The patient was living with her eldest son in a suburb in the vicinity of the hospital before the stroke occurred. She managed the household, did the housework and prepared their meals. Her hobbies included reading, knitting and walking for exercise.

\section{CLINICALEVALUATION}

The patient was evaluated by the second author who also treated her. Mrs X presented with expressive dysphasia and her short term memory (STM) appeared impaired. She needed assistance with feeding and had difficulty performing basic ADL

The patient's functional abilities were measured using the Barthel Index (BI) during the initial assessment, at discharge from hospital and at follow up as an outpatient (see table1: BI scores for Mrs X). The BI is a functional outcome measure developed in 1955 by Mahoney and Barthel to evaluate the ability of patients with neuromuscular and musculoskeletal disorders to care for themselves (Mahoney and Barthel, 1955).

The Barthel score primarily evaluates 10 activities of daily living: feeding, grooming, bathing, dressing, bowel function, bladder care, toilet use, ambulation, transfers, and stair climbing. The total score ranges from 0 to 100 (Sulter et al 1999, Stineman et.al 2003). The BI has shown acceptable internal consistency at admission and discharge, with a coefficient of $>0.71$ (D'Olhaberriague et al 1996, Sulter et al 1999). Hsueh, Lin, Jeng and Hsieh (2002) found the BI to be a reliable, valid, and responsive measure of basic ADL in patients with stroke. The authors chose the $\mathrm{BI}$ as an outcome measure because it is easy to use and has proven face value validity (Shah, Vanclay \& Cooper 1989; Wang 2006). The BI does not require special training or licensing to administer.

On admission the patient had a total BI score of 15 (see table1: BI scores for Mrs X). The patient's functional activities were affected, with mobility, feeding and bladder and bowel function being the worst affected. The functional activities were affected by the following impairments as per the findings of the physical evaluation:
$>$ decreased active movement of the right shoulder; decreased ability to place the arm and hold it in space.

$>$ poor dynamic balance in sitting and standing.

$>$ decreased ability to initiate and plan an activity/ movement; patient is unable to recall the sequence of movement.

$>$ decreased muscle tone in her right leg, therefore needs assistance with transfers and ambulation

$>\quad$ bladder and bowel incontinence

Table 1: Barthel Index Score for Mrs X.

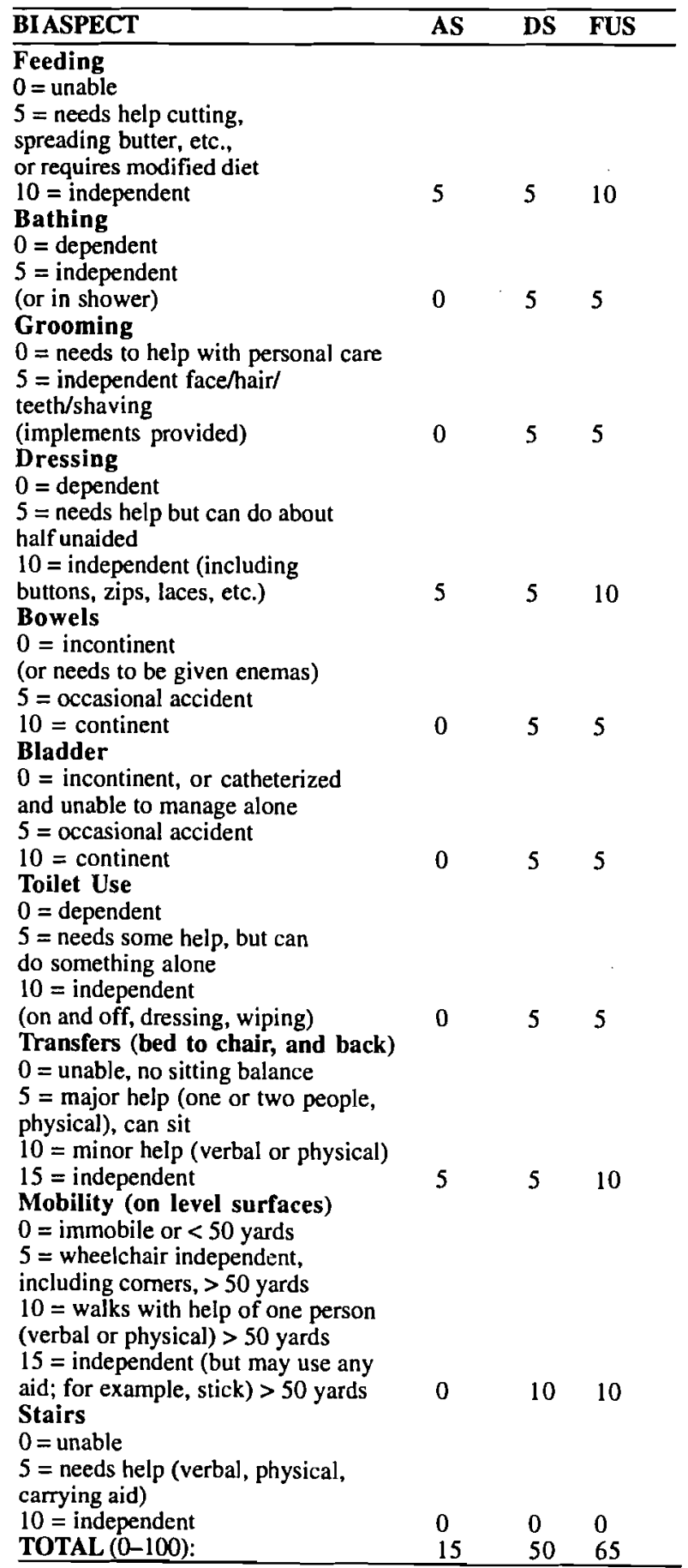

AS = Admission score; DS = Discharge score; FUS = Follow up score 


\section{INTERVENTION}

The rehabilitation programme was started on the day she was referred for physiotherapy. A team meeting was held with the patient, physiotherapist, the nursing staff and the patient's son to discuss short term rehabilitation goals for the patient and a plan of care. The patient's goal was to be able to walk without an aid inside the house and to use a cane/walking aid only when in public. The family's (patient and her son) desired outcome for her was, to be able to bath, feed and groom herself as well as dress and use the toilet without assistance. The patient's functional problems and impairments were taken into account during the team discussion and a comprehensive treatment plan was formulated to assist the patient to achieve these goals.

The team decided that in order to improve the patient's STM, a routine day program, with specific timeframes for different tasks and activities should be implemented. This would be repeated every day e.g. eating breakfast; washing and grooming activities; tea time; physiotherapy session; lunch; visit and exercise with her son; dinner; preparation for bedtime. The patient had two physiotherapy sessions per day. The activities that the patient performed in physiotherapy in the morning were taught to the patient's son who then repeated them in the afternoon. All the meals, dressing and grooming activities were supervised by the nursing staff.

To optimise the tone in her right arm and to improve the functional movement, task oriented activities were carried cut. The patient sat in front of a table, bearing weight through the right elbow which was supported on the table and read a book, paging through it with the unaffected left hand. Tlis was followed up by reaching for objects placed on the table by gliding the affected right arm on the surface of the table. The objects used were part of her grooming activities e.g. the hair brush and toothbrush. As part of the activity, the patient had to explain the function of each object and demonstrate its action with the left hand. At the end of the treatment session, the patient was asked questions on what she had read to improve her short term memory. Weight bearing to optimise motor control in the right lower extremity and dynamic balance exercises were done in standing. The patient stood in front of three tables of different heights with numbers on them. Task orientated activities in this position included moving object between the tables. This activity challenged her dynamic balance, weight transfer in standing, planning of movement and ability to remember and follow instructions.

Gait training was practised with a walking-frame. An obstacle course was set ùp and the patient had to plan her path to a specific destination. A reward awaited her at the other end of the course as an incentive to motivate the patient. Obstacles included a box - to walk around; a narrow space to walk through; and an uneven surface that she can only avoid by side stepping it.

To address the sequencing of movement, the patient repeatedly practised how to get up from supine lying to sitting over the side of the bed; dressing the upper and lower body and eating with the different forms of cutlery, with verbal guidance from the therapist and/or caregiver.

All of the above activities were repeated in the afternoon with her son, who was trained by the physiotherapist. This is too superficial. Please explain what the training of the son involved as well as the exact nature of his involvement, i.e. did he help her, support her physically or give her any other kind of encouragement? Good question, need to be specific and avoid unnecessary rhetoric.

The patient received a total of 10 treatments as an inpatient (two per day). Treatment was continued once a week on an outpatient basis for another four weeks. Two outpatient therapy sessions were cancelled as the patient was ill. When the patient was discharged, her son hired a caregiver to assist with the housework. The caregiver was also trained by the physiotherapist and given a checklist of activities to carry out as a home program. The home program was a replication of the physiotherapy program carried out in the hospital.

\section{OUTCOMES}

The patient's functional abilities were re-evaluated according to the BI scale after 4 weeks from discharge from hospital. The scores were compared to the initial and to discharge scores. The patient had a functional improvement of 35 points on the BI compared to her admission score. During the outpatient treatment period another 10 points were gained on the BI score and the patient scored a final total of 65 points. The short term goals which included the patient being able to feed independently and managing her grooming activities under supervision were also achieved. As this is a retrospective report of a clinical case, the first author, who was the patient's therapist, did all the assessments and treatment sessions.

\section{DISCUSSION}

The primary aim of this case report was to highlight the importance of family participation in the rehabilitation of a stroke patient. An inpatient rehabilitation programme was designed specifically to address the patient's needs and the family members (the patient's son mainly) were involved in the rehabilitation process. A very positive outcome was found and the patient's BI score improved from 20 on admission to 65 (this total does not match the above number) on the follow-up assessment after discharge from hospital.

\section{Use basic statistics to discuss outcome.}

Successful rehabilitation outcome is influenced by a number of factors including the extent of the brain injury, stroke survivor's attitude, family support and early rehabilitation. In the case of the patient under discussion, 
the latter two factors might have influenced the outcome.

\section{Family support}

The patient was part of a supportive family, and the members were actively involved in her rehabilitation. The positive attitude of the son is evidenced by his coming daily to hospital to treat the mother - presence at all treatment sessions, and eventually hiring and training a caregiver to continue the treatment. The authors are of the opinion that if her family did not support the rehabilitation programme, a different outcome may have been reached.

The importance of caregiver involvement from early on in the rehabilitation of stroke patients is well supported in the rehabilitation, literature (Hans and Haley 1999; Kaira et al 2004; Patel et al 2004 Visser-Meily, Post, Gorter, Van Berlekom, Van Den Boss \& Lindeman 2006). A study by Kaira et al (2004) found that training caregivers and involving them in the stroke patient's rehabilitation resulted in improved patient outcome. On the contrary, a study by Hale, Bennett, Bentley Crawshaw and Davis (2004) found that requiring too much involvement of the family members in the rehabilitation of a stroke survivor adds tremendous stress to the caregivers. The participants in the Hale et al study felt it was very demanding on the spouse to try to "turn them into a therapist" (Hale et al, 2004).

\section{Early rehabilitation}

The patient also received rehabilitation intervention early (within three days of sustaining the stroke). The establishment of a co-ordinated team effort for early evaluation and rehabilitation (within the first 48 hours after admission) can contribute to a shorter hospital stay, increased mobility and independence, and improve the quality of the family and patient's life (Hayes \& Carroll 1986).

Early intervention can help prevent secondary disabling conditions associated with stroke, positively influence functional activities and the ability to live independently (Van Peppen et al, 2004). A recent review of the literature on stroke rehabilitation by Diserens, Michel and Bogousslavsky (2007) suggests that early mobilisation is beneficial for stroke. Early rehabilitation helps patients to mobilise faster, thus contributing to improved outcomes after stroke (Paolucci, Antonucci and, Grasso 2000; Bernhardt, Diserens, Michel and Bogousslavsky 2008)

The patient was given a simple task oriented rehabilitation program which could be easily understood and followed through by the family members during the patients stay in hospital and after discharge. This ensured that functional gains achieved in rehabilitation will be maintained effectively through active family involvement (Evans et al. 1992, Maeshima 2003, Karla et al 2004).

According to the authors' observations, the patient appeared to have also improved in terms of memory and communication. The outcome measure used in this case report (BI) does not evaluate cognitive function, and therefore this aspect could not be objectively elaborated upon. Future reports of this nature should therefore use other scales which include a cognitive component like the Functional Index Measure (FIM).

\section{CONCLUSION}

This case report lends support to the literature that the involvement and training of family members in the rehabilitation of stroke patients has a potentially positive impact on rehabilitation outcome and subsequent quality of life. Although this case study cannot prove that family involvement was the main cause of the improved rehabilitation outcome of this patient, it highlights the important role of the family in the patient's rehabilitation. Further research on the effectiveness of family involvement on rehabilitation outcome is needed, preferably using randomised controlled trials.

\section{REFERENCES}

- Bernhardt J, Diserens K, Michel P, Bogousslavsky J (2008) Early mobilisation after stroke: Review of the literature. Cerebrovascular Disease. 24(1): 157-8.

- D’Olhaberriague L, Litvan I, Mitsas P, Mansbach HH (1996) A reappraisal of reliability and validity studies in stroke. Stroke 27: 2331-2336.

- Evans RL, Hendricks RD, Haselkorn, JK, Bishop DS, Baldwin D (1992). The family's role in stroke rehabilitation: a review of the literature. American Journal of Medical Rehabilitation 71:135-139.

- Galvin R, Cusack T and Stokes E (2008) 'To what extent are family members and friends involved in physiotherapy and the delivery of exercises to people with stroke?' Disability \& Rehabilitation, URL: http://dx.doi.org/10.1080/

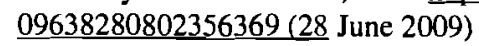

- Hale L, Bennett D, Bentley M, Crawshaw A, Davis H (2004). Stroke Rehabilitation - Comparing hospital and home-based physiotherapy: the patients' perception. New Zealand Journal of Physiotherapy 31(2): 84-92.

- Han B, Haley W (1999). Family care giving for patients with stroke. Stroke; 30:1478-5.

- Hayes SH, Carroll SR (1986). Early intervention care in acute stroke patient. Archive of Physical Medical Rehabilitation 67: 319-321.

- Hsueh IP, Lin JH, Jeng JS and Hsieh CL (2002) Comparison of the psychometric characteristics of the functional independence measure, 5 item Barthel index, and 10 item Barthel index in patients with stroke Journal of Neurology, Neurosurgery and Psychiatry; 73: 188-190.

- Kaira L, Evans A, Perez I, Melbourne A, Patel A, Donaldson N (2004) Training carers of stroke patients: randomized 
controlled trial. British Medical Journal 8:328:1009-1113.

- Kotila M, Numminen H, Waltimo O, Kaste M (1998). Depression after stroke: results of the FINNSTROKE study. Stroke 29: 368-372.

- Mahoney FI, Barthel DW (1955) "Functional evaluation: Barthel Index". Maryland State Medical Journal 14: 56-61.

- Stineman MG. Ross RN, Fiedler R, Granger CV, Maislin G (2003). Functional independence staging: conceptual foundation, face validity, and empirical derivation. Archives of Physical Medicine and Rehabilitation; 84: 29-37.

- Paolucci S, Antonucci G Grasso MG (2000). Early versus delayed inpatient stroke rehabilitation: a matched comparison conducted in Italy. Archives of Physical Medical Rehabilitation 81: 695-700.

- Patel A, Knapp M, Evans A, Perez I, Kaira L(2004) Training care givers of stroke patients: economic evaluation. British Medical Journal 328: 1102-1107.

- Shah S, Vanclay F and Cooper B (1989). Improving the sensitivity of the Barthel Index for stroke rehabilitation. Journal of Clinical Epidemiology 42(8): 703-709.
- Sulter G, Steen C, De Keyser J (1999) Use of the Barthel Index and the modified Ranking scale in acute stroke trials. Stroke 30: 1538-1541.

- Teraoka J,Burgard R (1992). Family support and stroke rehabilitation. The Western Journal of Medicine 157(6): 665666

- Van Peppen RP, Kwakkel G Wood-Dauphinee S, Hendriks HJ, Van der Wees PJ, Dekker J (2004). The impact of physical therapy on functional outcomes after stroke: What's the evidence? Clinical Rehabilitation.18: 833-862.

- Visser-Meily A, Post M, Gorter J-W, Van Berlekom SBY., Van Den Boss T and Lindeman E (2006). 'Rehabilitation of stroke patients needs a family-centred approach'. Disability \& Rehabilitation, 28:24, 1557-1561.

- Wang J (2006). Psychological abuse and its characteristic correlates among elderly Taiwanese. Archives of Gerontology and Geriatrics 42: 307-318.

- White CL, Lauzon S, Yaffe JM, Wood-Dauphinee S (2004). Towards a model of quality of life for family caregivers of stroke survivors. Quality of Life Research 13: 625-638. 